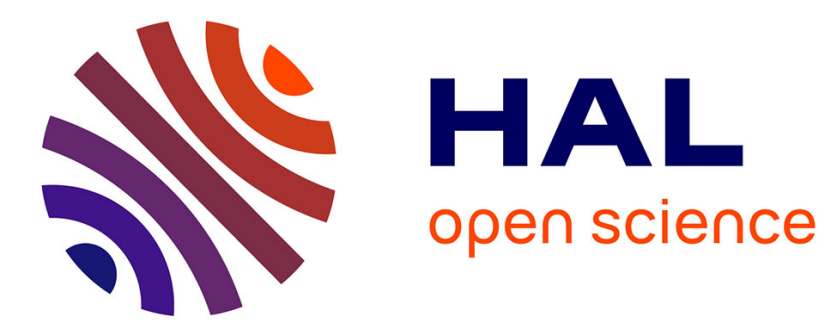

\title{
Study of propagation modes of bent waveguides and micro-ring resonators by means of the Aperiodic Fourier Modal Method
}

D. Bucci, B. Martin, A. Morand

\section{- To cite this version:}

D. Bucci, B. Martin, A. Morand. Study of propagation modes of bent waveguides and micro-ring resonators by means of the Aperiodic Fourier Modal Method. SPIE OPTO 2010, Jan 2010, San Francisco, CA, United States. pp.75970U, 10.1117/12.840686 . hal-00603655

\section{HAL Id: hal-00603655 \\ https://hal.science/hal-00603655}

Submitted on 17 Jan 2020

HAL is a multi-disciplinary open access archive for the deposit and dissemination of scientific research documents, whether they are published or not. The documents may come from teaching and research institutions in France or abroad, or from public or private research centers.
L'archive ouverte pluridisciplinaire HAL, est destinée au dépôt et à la diffusion de documents scientifiques de niveau recherche, publiés ou non, émanant des établissements d'enseignement et de recherche français ou étrangers, des laboratoires publics ou privés. 


\section{Study of propagation modes of bent waveguides and micro-ring resonators by means of the aperiodic Fourier modal method}

Bucci, Davide, Martin, Bruno, Morand, Alain

Davide Bucci, Bruno Martin, Alain Morand, "Study of propagation modes of bent waveguides and micro-ring resonators by means of the aperiodic Fourier modal method," Proc. SPIE 7597, Physics and Simulation of Optoelectronic Devices XVIII, 75970U (25 February 2010); doi: 10.1117/12.840686 


\title{
Study of propagation modes of bent waveguides and micro-ring resonators by means of the Aperiodic Fourier Modal Method
}

\author{
Davide Bucci, Bruno Martin, Alain Morand \\ IMEP - LAhC, Institut de Microélectronique, Électromagnétisme et Photonique, 3, parvis L. \\ Néel, 38016 Grenoble, France
}

\begin{abstract}
In the last years, several numerical methods have been studied and applied to the analysis of high index contrast bent waveguides. Very often, the problem is treated using a conformal mapping, which translates the bending into an equivalent graded index profile and a straight waveguide. In this article, we discuss the implementation of a full vectorial 2D mode solver by means of the Aperiodic Fourier Modal Method, developed directly in cylindrical coordinates. This does not require the conformal mapping technique. In the first part of our work, we develop a shorthand notation and the mathematical rules useful to describe the problem in a matrix form. The calculation of propagation modes is then reconducted to the search of eigenvectors of a matrix. We will at first confront our formulation in $1 \mathrm{D}$ with results described in the literature. In a second time, we will use the complete $2 \mathrm{D}$ solver to determine the resonance frequencies and the quality factors of micro-ring resonators made on silicon surrounded by silica. These characteristics are indeed related to the real and imaginary parts of the propagation constants. By comparison with 3D-FDTD analysis, we will show that our implementation can be used to accurately describe the behavior of micro-rings having a bending radius as low as $1.1 \mu \mathrm{m}$ in the near infrared region. This technique is general and can be applied to any micro-ring having an arbitrary cross-section and a quality factor which is less than 10000. Perspectives of this work include the study of the field propagation in a bent structure, as well as the coupling between micro-ring resonators and straight waveguides.
\end{abstract}

Keywords: Aperiodic Fourier Modal Method, Full Vectorial Mode Solver, Bent Waveguides, Micro-rings, RCWA

\section{INTRODUCTION}

One of the most important things to be considered while designing an integrated optical device is the knowledge of the propagation characteristics of the waveguides used in the design. For this reason, several numerical methods have been implemented, in order to study a more or less arbitrary wave-guiding structure. An interesting development is the Aperiodic Fourier Modal Method (AFMM). This technique has been proposed by Lalanne and Silberstein, ${ }^{1,2}$ in order to apply to waveguide structures the Rigorous Coupled Wave Method (RCWA), originally developed for the study of diffraction gratings. ${ }^{3}$ Thanks to the refinements proposed through the years, this method has been proved to be effective to study in a very general way waveguides made in different technologies. In this article, we will discuss a novel development of AFMM in cylindrical coordinates, for the study of bent waveguides. We will start in section 2 by describing our mathematical developments. Section 3 will be devoted to the comparison with the literature of the results given by our method, in some 1D test cases. Our formulation will then be used in section 4 to study the resonance properties of micro-rings obtained by silicon on silica technology in a complete 2D study. Our results will be again compared to the literature.

Further author information: (send correspondence to D. Bucci)

E-mail: bucci@minatec.grenoble-inp.fr, Telephone: +33 456529539

Physics and Simulation of Optoelectronic Devices XVIII, edited by

Bernd Witzigmann, Fritz Henneberger, Yasuhiko Arakawa, Marek Osinski, Proc. of SPIE

Vol. 7597, 75970U · (c) 2010 SPIE · CCC code: 0277-786X/10/\$18 · doi: 10.1117/12.840686

Proc. of SPIE Vol. 759775970 -1 


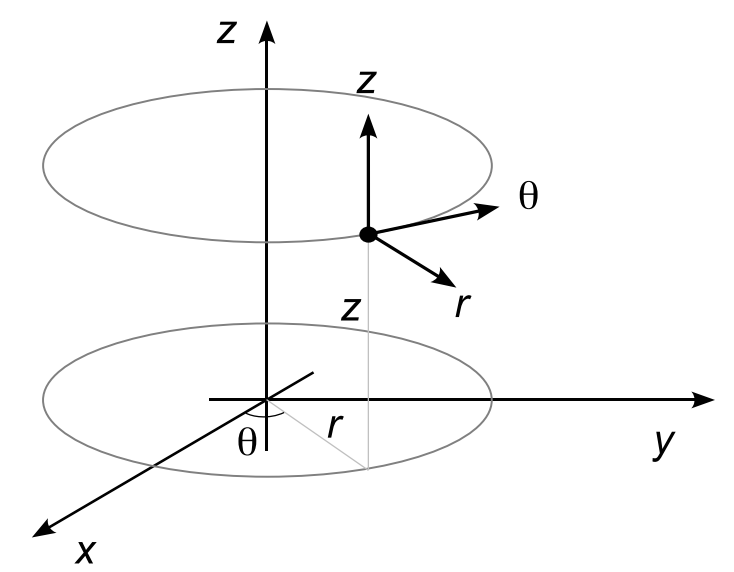

Figure 1. The cylindrical coordinate system used in the developments.

\section{THE DEVELOPMENT OF AFMM IN CYLINDRICAL COORDINATES}

In this section, we will see the mathematical background of our AFMM 2D mode solver, suited for bent waveguides. At first, we will manipulate Maxwell equations in cylindrical coordinates in order to describe the longitudinal evolution of transverse magnetic and electric fields in a bent waveguide. Then, we will develop a shorthand notation which will allow us to rewrite the equations in the Fourier space using a compact matrix notation. This will allow us to treat the modal problem as a search of eigenvalues and eigenvectors. The main advantage of the AFMM is that once we have all of them, we dispose of a complete set of guided as well as radiated propagation modes, which would allow us to treat also the propagation problem.

\subsection{Transverse fields calculation}

Let us consider the Maxwell equations in an uncharged anisotropic dielectric material, without sources. We study the harmonic behavior, for the electric and magnetic fields $\vec{E}$ and $\vec{H}$. Curl Maxwell equations can be written as follows, using the complex representation of $\vec{E}$ and $\vec{H}$ vectors:

$$
\left\{\begin{array}{r}
\nabla \wedge \vec{E}=-\mathrm{j} \omega \overrightarrow{\bar{\mu}} \vec{H} \\
\nabla \wedge \vec{H}=\mathrm{j} \omega \overline{\bar{\epsilon}} \vec{E}
\end{array}\right.
$$

where $\omega$ is the time radian frequency of the fields, $\overline{\bar{\epsilon}}$ and $\overline{\bar{\mu}}$ are respectively the diagonal tensors of permittivity and permeability:

$$
\begin{aligned}
\overline{\bar{\epsilon}} & =\left(\begin{array}{ccc}
\epsilon_{r} & 0 & 0 \\
0 & \epsilon_{z} & 0 \\
0 & 0 & \epsilon_{\theta}
\end{array}\right) \\
\overline{\bar{\mu}} & =\left(\begin{array}{ccc}
\mu_{r} & 0 & 0 \\
0 & \mu_{z} & 0 \\
0 & 0 & \mu_{\theta}
\end{array}\right)
\end{aligned}
$$

Our goal is to calculate the propagation modes of waveguides, by taking into account the effect of a bend of constant radius. The propagation will thus been along $\theta$. Our version of the Aperiodic Fourier Modal Method is based on the definition of a propagation operator, derived from the Maxwell equations in the Fourier space. We use a cylindrical coordinate system instead of a cartesian one, as shown in figure 1.

By developing the different components the curl Maxwell equations(1), they can be rewritten as a set of 6 equations describing the field $\left(E_{r}, E_{\theta}, E_{z}\right.$ and $\left.H_{r}, H_{\theta}, H_{z}\right)$. The longitudinal components $E_{\theta}$ and $H_{\theta}$ can be calculated from the other field components, injected in the remaining four equations and the field derivatives 


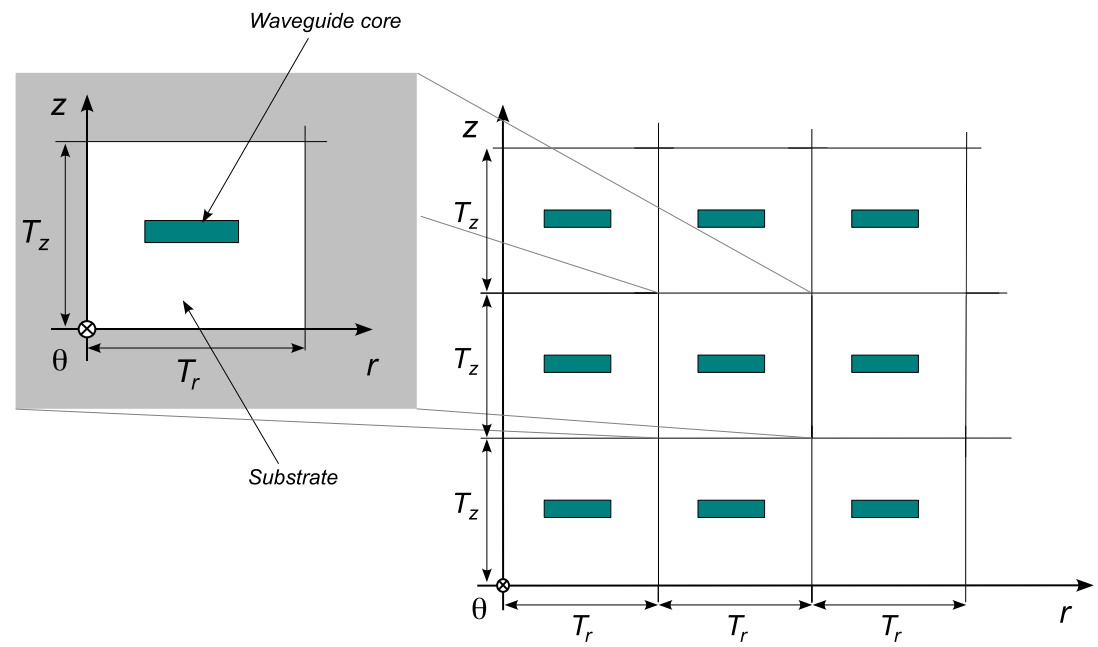

Figure 2. The calculation window and the effect of the periodization applied to develop fields, permittivities and permeabilities in Fourier series.

versus $\theta$ can be made explicit:

$$
\left\{\begin{array}{rrr}
\frac{\partial E_{r}}{\partial \theta}= & \mathrm{j} \omega \mu_{z} r H_{z}+\frac{1}{\mathrm{j} \omega} \frac{1}{\epsilon_{\theta}}\left(\frac{\partial H_{r}}{\partial z}-\frac{\partial H_{z}}{\partial r}\right)+\frac{1}{\mathrm{j} \omega} r \frac{\partial}{\partial r}\left(\frac{1}{\epsilon_{\theta}}\right)\left(\frac{\partial H_{r}}{\partial z}-\frac{\partial H_{z}}{\partial r}\right)+\frac{1}{\mathrm{j} \omega} \frac{r}{\epsilon_{\theta}}\left(\frac{\partial^{2} H_{r}}{\partial r \partial z}-\frac{\partial^{2} H_{z}}{\partial r^{2}}\right) \\
\frac{\partial E_{z}}{\partial \theta}= & -\mathrm{j} \omega \mu_{r} r H_{r}+\frac{1}{\mathrm{j} \omega} r \frac{\partial}{\partial z}\left(\frac{1}{\epsilon_{\theta}}\right)\left(\frac{\partial H_{r}}{\partial z}-\frac{\partial H_{z}}{\partial r}\right)+\frac{1}{\mathrm{j} \omega} r \frac{1}{\epsilon_{\theta}}\left(\frac{\partial^{2} H_{r}}{\partial z^{2}}-\frac{\partial^{2} H_{z}}{\partial z \partial r}\right) \\
\frac{\partial H_{r}}{\partial \theta}= & -\mathrm{j} \omega \epsilon_{z} r E_{z}-\frac{1}{\mathrm{j} \omega} \frac{1}{\mu_{\theta}}\left(\frac{\partial E_{r}}{\partial z}-\frac{\partial E_{z}}{\partial r}\right)-\frac{1}{\mathrm{j} \omega} r \frac{\partial}{\partial r}\left(\frac{1}{\mu_{\theta}}\right)\left(\frac{\partial E_{r}}{\partial z}-\frac{\partial E_{z}}{\partial r}\right)-\frac{1}{\mathrm{j} \omega} \frac{r}{\mu_{\theta}}\left(\frac{\partial^{2} E_{r}}{\partial r \partial z}-\frac{\partial^{2} E_{z}}{\partial r^{2}}\right) \\
\frac{\partial H_{z}}{\partial \theta}= & \mathrm{j} \omega \epsilon_{r} r E_{r}-\frac{1}{\mathrm{j} \omega} r \frac{\partial}{\partial z}\left(\frac{1}{\mu_{\theta}}\right)\left(\frac{\partial E_{r}}{\partial z}-\frac{\partial E_{z}}{\partial r}\right)-\frac{1}{\mathrm{j} \omega} r \frac{1}{\mu_{\theta}}\left(\frac{\partial^{2} E_{r}}{\partial z^{2}}-\frac{\partial^{2} E_{z}}{\partial z \partial r}\right)
\end{array}\right.
$$

The equations (4) represent the differential field propagation operator on the $\theta$ axis.

\subsection{Fourier series field development}

The AFMM consists in applying an artificial periodicity to the studied structure. This allows us to develop in Fourier series all fields, permeabilities and permittivities appearing in the equations. We will consider the case of a $\theta$-invariant structure, such as a waveguide having a constant bending radius or a micro-ring resonator. Thus, the Fourier developments will be done on the plane transverse to the propagation direction: $r$ and $z$. This is shown in figure 2, which also depicts the effect of the periodization. If the periods $T_{r}=2 \pi / \nu_{r}$ and $T_{z}=2 \pi / \nu_{z}$ are sufficiently long, we can expect the results of the calculations be representative even for the non-periodic waveguide.

The artificial periodization allows us to rewrite equations 4, by developing each term as a sum of Fourier space harmonics in the transverse plane. This means that a generic $A$ term can be represented as follows:

$$
A=\sum_{h} \sum_{k} a_{h, k} \mathrm{e}^{\mathrm{j}\left(h \nu_{r} r+k \nu_{z} z\right)}
$$

where $h$ and $k$ are integers, $a_{h, k}$ is the $h$-order along $r$ and $k$-order along $z$ harmonic; $\nu_{r}$ and $\nu_{z}$ are the fundamental frequencies along $r$ and $z$. Equations 4 contains several products between terms which are developed in Fourier series. This leads to convolutions in the Fourier space, complicated by the fact that they sometimes involve derivatives. Three points have to be discussed:

1. We need a way to represent the convolutions in the Fourier space, as products between a matrix and a vector. This implies that we need to truncate the Fourier development to a finite number of harmonics. 
2. We need to take into account in the same way the convolutions between multiple terms, which appear when taking into account the term $r$, which is present in equations 4 .

3. We have to develop a shorthand notation to represent derivatives in a consistent way.

\subsection{The modified block-Toeplitz matrix}

We will call $S_{r}$ and $S_{z}$ the number of harmonics taken in the $r$ and $z$ dimension. A product between two generic terms $M$ and $C$ in the original space thus becomes a truncated convolution:

$$
t_{i, l}=\sum_{h=-S_{r}}^{S_{r}} \sum_{k=-S_{z}}^{S_{z}} m_{i-h, l-k} c_{h, k}
$$

which can be written on the form of a product between a Toeplitz matrix and a column vector:

$$
T=\llbracket M \rrbracket[C]
$$

In $1 \mathrm{D}, \llbracket M \rrbracket$ is obtained by building a Toeplitz matrix from the Fourier components $m_{i, l}$. In $2 \mathrm{D}$, we need to unroll the $r$ and $z$ components of the $c_{h, k}$ space harmonics in order to write the vector $[C]$ by aligning one column after the other. Doing this allows us to write matrix $\llbracket M \rrbracket$ from $m_{i, l}$ as a block-Toeplitz matrix. In the rest of the article, we will use the notation $\llbracket M \rrbracket$ to stress that this is a block-Toeplitz matrix built from the $m_{i, l}$ Fourier coefficients:

$$
\llbracket M \rrbracket=\left(\begin{array}{cccc}
\overline{\bar{M}}_{0} & \overline{\bar{M}}_{-1} & \overline{\bar{M}}_{-2} & \cdots \\
\overline{\bar{M}}_{1} & \overline{\bar{M}}_{0} & \overline{\bar{M}}_{-1} & \cdots \\
\overline{\bar{M}}_{2} & \overline{\bar{M}}_{1} & \overline{\bar{M}}_{0} & \cdots \\
\cdots & & &
\end{array}\right)
$$

in which each block $\overline{\bar{M}}_{l}$ can be written as follows:

$$
\overline{\bar{M}}_{l}=\left(\begin{array}{cccc}
m_{0, l} & m_{-1, l} & m_{-2, l} & \cdots \\
m_{1, l} & m_{0, l} & m_{-1, l} & \cdots \\
m_{2, l} & m_{1, l} & m_{0, l} & \cdots \\
\cdots & & &
\end{array}\right)
$$

The size of the (square) $\llbracket M \rrbracket$ matrix is thus $\left[\left(S_{r}+1\right)\left(S_{z}+1\right)\right] \times\left[\left(S_{r}+1\right)\left(S_{z}+1\right)\right]$. $^{*}$ We will sometimes also use the notation $[C]_{h, k}$ instead of $c_{h, k}$.

\subsection{Representing derivatives}

In equation (4), we can find also more complex products, like the following term:

$$
R=\frac{\partial}{\partial z}\left(\frac{1}{\epsilon_{\theta}}\right) \frac{\partial H_{z}}{\partial r}
$$

By developing in Fourier series each term and the derivatives, to calculate the term $[R]_{i, l}$ we obtain a convolution in which we have a multiplication of $\left[H_{z}\right]_{h, k}$ by $\mathrm{j} h \nu_{r}$. This is coming from the $r$ derivative of $H_{z}$. We obtain also the multiplication of $\left[1 / \epsilon_{\theta}\right]_{i-h, l-k}$ by the term $\mathrm{j}(l-k) \nu_{z}$, which comes from the $z$ derivative of $\epsilon_{\theta}^{-1}$. We can again write this convolution as a matrix by vector product:

$$
R=\mathrm{j}^{2} \llbracket \frac{1}{\epsilon_{\theta}} \rrbracket_{z}^{(r)}\left[H_{z}\right]
$$

where the $\left[H_{z}\right]$ vector is built as usual from the unrolled $H_{z}$ Fourier components and $\mathrm{j}^{2}=-1$ comes from the double derivative. The shorthand notation $\llbracket 1 / \epsilon_{\theta} \rrbracket_{z}^{(r)}$ means that the matrix takes into account the products

\footnotetext{
${ }^{*}$ If we take $S_{r}$ space harmonics, this gives us $2 S_{r}+1$ Fourier coefficients. The convolution gives us $S_{r}+1$ Fourier terms in the result, thus giving a matrix whose size is $\left[\left(S_{r}+1\right)\left(S_{z}+1\right)\right] \times\left[\left(S_{r}+1\right)\left(S_{z}+1\right)\right]$.
} 
coming from the derivatives. This can be obtained directly by using the Hadamard product of the above described bloc-Toeplitz matrix with two matrices $\mathcal{C}_{z}$ and $\mathcal{C}^{(r)}$, containing the multiplication terms:

$$
\llbracket \frac{1}{\epsilon_{\theta}} \rrbracket_{z}^{(r)}=\llbracket \frac{1}{\epsilon_{\theta}} \rrbracket \bullet \mathcal{C}_{z} \bullet \mathcal{C}^{(r)}
$$

The first matrix $\mathcal{C}_{z}$ is the block-Toeplitz matrix built with space harmonics:

$$
\mathcal{C}_{z}=\left(\begin{array}{cccc}
C_{0} & C_{-1} & C_{-2} & \cdots \\
C_{1} & C_{0} & C_{-1} & \cdots \\
C_{2} & C_{1} & C_{0} & \cdots \\
\cdots & & &
\end{array}\right)
$$

in which each block $C_{l}$ is a constant matrix:

$$
C_{l}=l \nu_{z}\left(\begin{array}{cccc}
1 & 1 & 1 & \ldots \\
1 & 1 & 1 & \ldots \\
1 & 1 & 1 & \ldots \\
\cdots & & &
\end{array}\right)
$$

The matrix $\mathcal{C}^{(r)}$ is made by $\left(S_{r}+1\right)\left(S_{z}+1\right)$ identical columns, containing multiples of the fundamental space

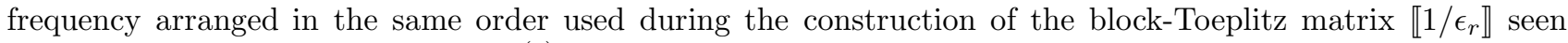
above. We will call the matrix $\llbracket 1 / \epsilon_{r} \rrbracket_{z}^{(r)}$ a modified bloc-Toeplitz matrix, obtained from the Fourier development of $1 / \epsilon_{\theta}$. To represent in the truncated Fourier space products and derivatives, one has to write the corresponding modified bloc-Toeplitz matrix. The same principle can be applied to all terms.

\subsection{Products of several terms}

Because of the periodization and the curl expressiond in cylindrical coordinates, the evolution of the bending radius $r$ in equations appears like a sawtooth wave, which has to be multiplied to several terms. Its Fourier $n$-th order harmonics $[r]_{n}$ can be calculated analytically:

$$
[r]_{n}= \begin{cases}r_{0} & \text { if } n=0 \\ \mathrm{j} \frac{T_{r}}{2 \pi n} & \text { if } n \neq 0 .\end{cases}
$$

where $r_{0}$ and $T_{r}$ are respectively the bending radius of the center and the width of the calculation window. The $\llbracket r \rrbracket$ matrix will thus be the bloc-Toeplitz matrix containing the harmonics given by equation (15). It can be shown that a product of several terms in the original space leads to the product of Toeplitz matrices in the Fourier space, if we tolerate an error which comes from the missing harmonics in the convolution. ${ }^{4}$

\subsection{The propagation operator in matrix form}

The rules described above allow us to write the equations 4 in matrix form. The propagation along $\theta$ is a phase shift term:

$$
[F]=[\hat{F}] \mathrm{e}^{-\mathrm{j} \beta_{\mathrm{s}} \theta}
$$

where $F$ can be either $E_{r}, E_{z}, H_{r}, H_{z}$. We can thus rewrite equations 4 as follows:

$$
-\mathrm{j} \beta_{\mathrm{s}}\left(\begin{array}{c}
{\left[\hat{E}_{r}\right]} \\
{\left[\hat{E}_{z}\right]} \\
{\left[\hat{H}_{r}\right]} \\
{\left[\hat{H}_{z}\right]}
\end{array}\right)=\frac{1}{\mathrm{j} \omega} \overline{\bar{A}}\left(\begin{array}{c}
{\left[\hat{E}_{r}\right]} \\
{\left[\hat{E}_{z}\right]} \\
{\left[\hat{H}_{r}\right]} \\
{\left[\hat{H}_{z}\right]}
\end{array}\right)
$$

where:

$$
\overline{\bar{A}}=\left(\begin{array}{cccc}
0 & 0 & \overline{\bar{X}}_{1} & \overline{\bar{X}}_{2} \\
0 & 0 & \overline{\bar{X}}_{3} & \overline{\bar{X}}_{4} \\
\overline{\bar{Y}}_{1} & \overline{\bar{Y}}_{2} & 0 & 0 \\
\overline{\bar{Y}}_{3} & \overline{\bar{Y}}_{4} & 0 & 0
\end{array}\right)
$$


A direct application of the rules described above for $\overline{\bar{X}}_{i}$ and $\overline{\bar{Y}}_{i}, i \in\{1,2,3,4\}$, gives an unsatisfying convergence for a discontinuous field. This problem has long been believed to be a consequence of Gibbs phenomena occurring at discontinuities. In 1996, Lalanne and Morris ${ }^{5}$ reported new development rules which greatly enhanced the convergence in this case. The mathematical context has been explored by $\mathrm{Li}^{6}$ in the same year. TM and TE fields can be studied separately in the $1 \mathrm{D}$, but in $2 \mathrm{D}$ the field components are coupled. ${ }^{7}$ For that reason, it is not straightforward to study the $2 \mathrm{D}$ case, since one does not know a priori which field components are discontinuous. We used the following development:

$$
\begin{array}{lr}
\overline{\bar{X}}_{1}= & \mathrm{j} \llbracket \epsilon_{\theta} \rrbracket^{-1,(z)}-\llbracket r \rrbracket\left(\llbracket \epsilon_{\theta} \rrbracket_{r}^{-1,(z)}+\llbracket \epsilon_{\theta} \rrbracket^{-1,(r z)}\right) \\
\overline{\bar{X}}_{2}= & -\mathrm{j} \llbracket \epsilon_{\theta} \rrbracket^{-1,(r)}+\llbracket r \rrbracket\left(-\omega^{2} \llbracket \mu_{z} \rrbracket+\llbracket \epsilon_{\theta} \rrbracket_{r}^{-1,(r)}+\llbracket \epsilon_{\theta} \rrbracket^{-1,(r r)}\right) \\
\overline{\bar{X}}_{3}= & \llbracket r \rrbracket\left(\omega^{2} \llbracket \mu_{r} \rrbracket+\llbracket \epsilon_{\theta} \rrbracket_{z}^{-1,(z)}-\llbracket \epsilon_{\theta} \rrbracket^{-1,(z z)}\right) \\
\overline{\bar{X}}_{4}= & \llbracket r \rrbracket\left(\llbracket \epsilon_{\theta} \rrbracket_{z}^{-1,(r)}+\llbracket \epsilon_{\theta} \rrbracket^{-1,(z r)}\right) \\
\overline{\bar{Y}}_{1}= & -\mathrm{j} \llbracket \mu_{\theta} \rrbracket^{-1,(z)}+\llbracket r \rrbracket\left(\llbracket \mu_{\theta} \rrbracket_{r}^{-1,(z)}+\llbracket \mu_{\theta} \rrbracket^{-1,(r z)}\right) \\
\overline{\bar{Y}}_{2}= & \mathrm{j} \llbracket \mu_{\theta} \rrbracket^{-1,(r)}+\llbracket r \rrbracket\left(\omega^{2} \llbracket \epsilon_{z} \rrbracket-\llbracket \mu_{\theta} \rrbracket_{r}^{-1,(r)}-\llbracket \mu_{\theta} \rrbracket^{-1,(r r)}\right) \\
\overline{\bar{Y}}_{3}= & \llbracket r \rrbracket\left(-\omega^{2} \llbracket \epsilon_{r} \rrbracket+\llbracket \mu_{\theta} \rrbracket_{z}^{-1,(z)}+\llbracket \mu_{\theta} \rrbracket^{-1,(z z)}\right) \\
\overline{\bar{Y}}_{4}= & -\llbracket r \rrbracket\left(\llbracket \mu_{\theta} \rrbracket_{z}^{-1,(r)}+\llbracket \mu_{\theta} \rrbracket^{-1,(z r)}\right)
\end{array}
$$

In other words, instead of using the matrix $\llbracket 1 / \epsilon_{\theta} \rrbracket$, we took $\llbracket \epsilon_{\theta} \rrbracket^{-1}$ (and the same for permeabilities), which gives exactly the same result for an infinite number of harmonics, ${ }^{4}$ but significantly increase the convergence rate in the truncated case.

In this formulation, the modes supported by the structure are the eigenvectors of the $\overline{\bar{A}}$ matrix. The associated propagation constants $\beta_{\mathrm{s}}$ are the corresponding eigenvalues. While in the rectangular case it is straightforward to define a propagation effective index, this definition in cylindrical coordinates is somewhat arbitrary. Our choice has been to normalize the propagation constant on $r_{0}$, the bending radius of the center of the calculation window. Our choice thus leads to the following definition of the effective index of bent waveguides:

$$
n_{\mathrm{eff}}=\frac{\beta_{\mathrm{s}}}{r_{0} k_{0}}
$$

where $k_{0}=2 \pi / \lambda$ is the wave number in the vacuum.

\subsection{Perfectly matched layers}

A practical consequence of the periodization described in section 2.2 is that we cannot consider each single structure comprised in the calculation window as completely independent from its copies, due to the periodization. For this reason, the introduction of Perfectly Matched Layers has been proposed in order to reduce (or ideally eliminate) the influence of each repetition of the calculation window.

The method we used has been proposed by S.J. Hewlett and F. Ladouceur ${ }^{8}$ and extended by J.P. Hugonin and P. Lalanne ${ }^{9,10}$ for the case of a vectorial mode solver. The idea consists in using a nonlinear coordinate transform in order to map the finite calculation window in an infinite region. The main advantages of this technique consist in achieving a better extinction of the field far from the waveguide, thus allowing to obtain useful results even with a smaller calculation window in comparison with the anisotropic PMLs as described for example by Silberstein et al. ${ }^{2}$ The application of efficient PMLs in the calculation window is important in our case, since they are responsible for the absorption of the radiated field due to the bending.

In our calculations, we used the coordinate transform proposed by J.P. Hugonin and P. Lalanne. ${ }^{10}$ 


\begin{tabular}{c|ccl|ccc}
\hline \multirow{2}{*}{$\begin{array}{c}\text { Radius } \\
(\mu \mathrm{m})\end{array}$} & \multicolumn{3}{|c|}{ real part of $\beta_{\mathrm{s}}$} & \multicolumn{3}{c}{ imaginary part of $\beta_{\mathrm{s}}$} \\
\cline { 2 - 7 } & Hiremath $^{11}$ & AFMM (conf.) & AFMM (cyl.) & Hiremath ${ }^{11}$ & AFMM (conf.) & AFMM (cyl.) \\
\hline 50 & $4.0189 \times 10^{2}$ & $4.0189 \times 10^{2}$ & $4.0189 \times 10^{2}$ & $-7.9973 \times 10^{-2}$ & $-7.9963 \times 10^{-2}$ & $-7.9999 \times 10^{-2}$ \\
100 & $8.0278 \times 10^{2}$ & $8.0278 \times 10^{2}$ & $8.0278 \times 10^{2}$ & $-9.6030 \times 10^{-4}$ & $-9.6008 \times 10^{-4}$ & $-9.6364 \times 10^{-4}$ \\
150 & $1.2039 \times 10^{3}$ & $1.2039 \times 10^{3}$ & $1.2039 \times 10^{3}$ & $-7.3910 \times 10^{-6}$ & $-1.0577 \times 10^{-5}$ & $-1.0004 \times 10^{-5}$ \\
200 & $1.6051 \times 10^{3}$ & $1.6051 \times 10^{3}$ & $1.6051 \times 10^{3}$ & $-4.8965 \times 10^{-8}$ & $-1.0743 \times 10^{-7}$ & $-2.1264 \times 10^{-7}$ \\
\hline
\end{tabular}

Table 1. TE $\mathrm{T}_{0}$ propagation constants for medium refractive index contrast confinement with different bend radius $r_{0}$ and parameters $\left(n_{\mathrm{s}}=1.6, n_{\mathrm{c}}=1.7, n_{\mathrm{s}}=1.6\right)$, width $w=1 \mu \mathrm{m}$ and wavelength $\lambda=1.3 \mu \mathrm{m}$.

\section{APPLICATION TO THE 1D CASE}

Before considering the general 2D case, we test our method in 1D. This allows us to compare its behavior versus the results given by other scalar methods, such as the one proposed by Hiremath et al. ${ }^{11}$ as well as the standard conformal mapping technique. ${ }^{12}$

As a brief description, the method proposed by Hiremath et al. ${ }^{11}$ is similar to the multilayer method applied in cylindrical coordinates. Fields are expressed in terms of Bessel $\left(\mathrm{J}_{\beta_{\mathbf{s}}}(a)\right.$ and $\left.\mathrm{Y}_{\beta_{\mathbf{s}}}(a)\right)$ and Hankel $\left(\mathrm{H}_{\beta_{\mathbf{s}}}^{(1)}(a)\right.$ and $\mathrm{H}_{\beta_{\mathrm{s}}}^{(2)}(a)$ ) functions of complex order $\beta_{\mathrm{s}}$ (which corresponds to our propagation constant towards $\theta$ ) and real argument $a$. Considering a non-divergent solution inside the bend and only an outgoing wave $\left(\mathrm{H}_{\beta_{\mathrm{s}}}^{(2)}(a)\right)$ outside the bend, the system leads to a homogeneous system. The propagation constants $\beta_{\mathrm{s}}$ are determined from the zeros of the determinant of this system.

On the other hand, the standard conformal mapping technique ${ }^{12}$ consists instead in applying the AFMM method in a conformal map. ${ }^{13}$ Indeed, a bent waveguide in a cylindrical coordinate system corresponds to a straight waveguide in the conformal map. The conformal map is represented by the (u,v) plane:

$$
u=r_{0} \ln \left(\frac{r}{r_{0}}\right), v=r_{0} \theta
$$

where $r_{0}$ is the radius of curvature and the center of the bent waveguide. This transformation involves a modification of the refractive index contrast distribution, in order to keep the leaking aspect of the structure. This modification consists of a simple multiplication of the actual refractive index with an exponential function depending on $r_{0}$.

These three methods are able to compute the propagation constants $\beta_{\mathrm{s}}$ and the field profiles that characterize the modes of a waveguide. Thereby, we have chosen this quantity as a criterion.

We consider two types of confinement studied by Hiremath et al. ${ }^{11}$ for the comparison. In the two cases, the width of the waveguide core $w$ is set to $1 \mu \mathrm{m}$ and the radius $r_{0}$ corresponds to the middle of the waveguide. The wavelength $\lambda$ is set to $1.3 \mu \mathrm{m}$. In the first case, corresponding to a medium confinement, the substrate has a refractive index $n_{\mathrm{s}}$ set to 1.6 and the bent waveguide core has a refractive index $n_{\mathrm{c}}$ set to 1.7. The radii $r_{0}$ range from $50 \mu \mathrm{m}$ to $200 \mu \mathrm{m}$. In the second case, corresponding to a low confinement, the substrate has a refractive index $n_{\mathrm{s}}$ set to 3.22 and the bent waveguide core has a refractive index $n_{\mathrm{c}}$ set to 3.26106 . The radii $r_{0}$ go from $200 \mu \mathrm{m}$ to $1000 \mu \mathrm{m}$.

We have chosen a simulation window of $20 \mu \mathrm{m}$, with $2 \mu \mathrm{m}$ width PMLs having a $\gamma$ coefficient of $0.5-\mathrm{j} 0.5$ at each side and $S_{\mathrm{r}}=100$ harmonics. As we consider the 1D case, only the fundamental harmonic is used in z-axis. These parameters have been set at the same values in the conformal mapping along the u-axis. ${ }^{12}$

Tables 1 and 2 give the propagation constants for the two test cases described above.

From these tables, it can be seen that the three methods give very close results $\left(10^{-4}\right)$ concerning the real parts of the propagation constants. For low radii, the imaginary parts are similar with less than 1 percent of relative difference. For large radii, the AFMM over-estimates these values. Indeed, in this case, the influence of the PMLs is more preponderant than the bend. 


\begin{tabular}{c|ccl|ccc}
\hline \multirow{2}{*}{$\begin{array}{c}\text { Radius } \\
(\mathrm{\mu m})\end{array}$} & \multicolumn{3}{|c|}{ real part of $\beta_{\mathrm{S}}$} & \multicolumn{3}{c}{ imaginary part of $\beta_{\mathrm{S}}$} \\
\cline { 2 - 7 } & Hiremath $^{11}$ & AFMM (conf.) & AFMM (cyl.) & Hiremath $^{11}$ & AFMM (conf.) & AFMM (cyl.) \\
\hline 200 & $3.1364 \times 10^{3}$ & $3.1364 \times 10^{3}$ & $3.1364 \times 10^{3}$ & $-6.2135 \times 10^{-1}$ & $-6.2118 \times 10^{-1}$ & $-6.2159 \times 10^{-1}$ \\
400 & $6.2700 \times 10^{3}$ & $6.2700 \times 10^{3}$ & $6.2700 \times 10^{3}$ & $-4.9158 \times 10^{-2}$ & $-4.9226 \times 10^{-2}$ & $-4.9233 \times 10^{-2}$ \\
600 & $9.4041 \times 10^{3}$ & $9.4041 \times 10^{3}$ & $9.4041 \times 10^{3}$ & $-2.5635 \times 10^{-3}$ & $-2.5588 \times 10^{-3}$ & $-2.5610 \times 10^{-3}$ \\
800 & $1.2538 \times 10^{4}$ & $1.2538 \times 10^{4}$ & $1.2538 \times 10^{4}$ & $-1.1174 \times 10^{-4}$ & $-1.1625 \times 10^{-4}$ & $-1.1626 \times 10^{-4}$ \\
1000 & $1.5673 \times 10^{4}$ & $1.5673 \times 10^{4}$ & $1.5673 \times 10^{4}$ & $-4.4742 \times 10^{-6}$ & $-1.1718 \times 10^{-5}$ & $-1.1466 \times 10^{-5}$ \\
\hline
\end{tabular}

Table 2. $\mathrm{TE}_{0}$ propagation constants for low refractive index contrast confinement with different bend radius $r_{0}$ and parameters $\left(n_{\mathrm{s}}=3.22, n_{\mathrm{c}}=3.26106, n_{\mathrm{s}}=3.22\right)$, width $w=1 \mu \mathrm{m}$ and wavelength $\lambda=1.3 \mu \mathrm{m}$.

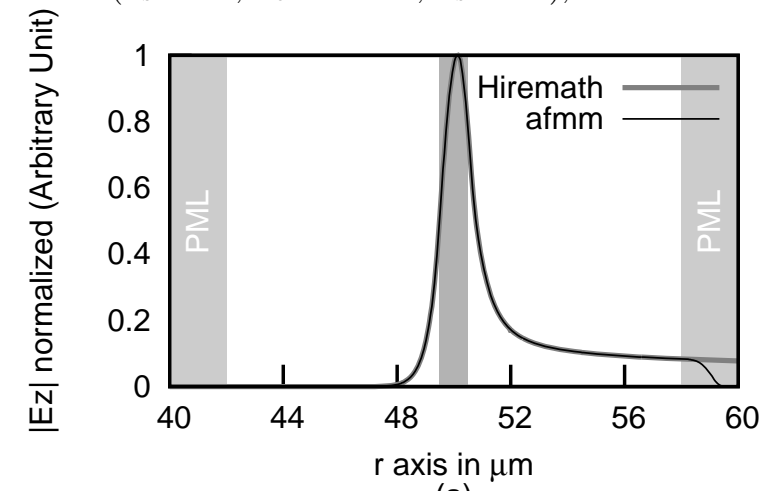

(a)

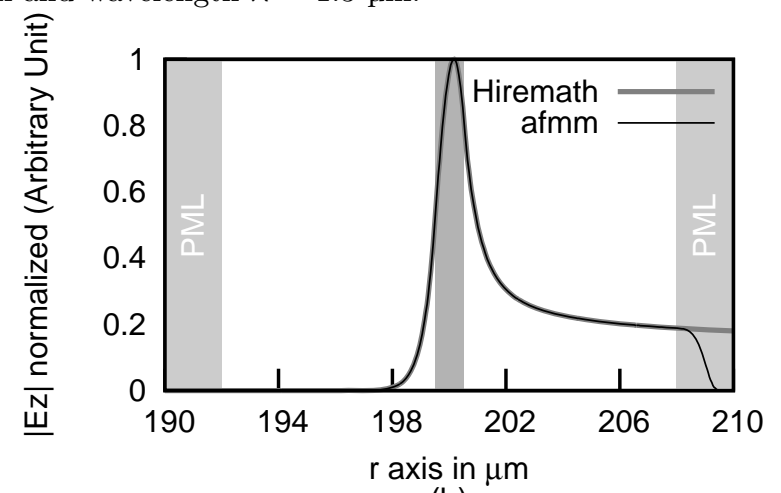

(b)

Figure 3. Module of $\mathrm{TE}_{0} \mathrm{Ez}$ fields component computed by the analytical method and by the AFMM for the medium contrast (a) and low contrast (b) structures

Figure 3 shows the field representation of the $\mathrm{TE}_{0}$ mode of the medium contrast waveguide with a radius of $50 \mu \mathrm{m}$ and the $\mathrm{TE}_{0}$ mode of the low contrast waveguide with a radius of $200 \mu \mathrm{m}$.

From these plots, the great similitude of the representations can be noticed (less than $0.1 \%$ of relative amplitude difference). The effect of the PMLs can also be noticed at the edge of the simulation window where the field is indeed attenuated.

We can point out that a minimum number of harmonics is needed to obtain a suitable propagation constant and a stable field representation. With 60 harmonics, the real part of the propagation constant keep quietly close $\left(10^{-3}\right)$ and the imaginary part are similar with about 3 percent of relative difference. With less than 60 harmonics, oscillations due to the PMLs appear on the left part of the mode profile.

\section{THE FULL VECTORIAL 2D CASE: APPLICATION TO MICRO RINGS}

Given the encouraging results obtained in the 1D case, to test the behavior of our full vectorial mode solver, we consider now the study of a micro-ring shaped resonator. The properties of such a structure are very interesting for a number of applications such as lasers ${ }^{14}$ and add \& drop devices. ${ }^{15}$ Our results will be compared with the 3D-FDTD results published by of Armaroli et al. ${ }^{16}$ We thus consider a silicon micro-ring $\left(n_{\mathrm{c}}=3.48\right)$ immersed in silica $\left(n_{\mathrm{s}}=1.44\right)$. Its thickness is $300 \mathrm{~nm}$, the internal and external bending radii are respectively $1 \mu \mathrm{m}$ and $1.2 \mu \mathrm{m}$.

The real part of the complex effective index $n_{\text {eff }}$ calculated for a guided mode is related to the azimuthal order $m$ at a given wavelength $\lambda$ :

$$
m=\frac{2 \pi}{\lambda} r_{0} \mathbb{R}\left\{n_{\mathrm{eff}}\right\}
$$

where $r_{0}$ is the bending radius of the center of our calculation window. If the azimuthal order $m$ is an integer value, we do have a resonance at that particular wavelength. The quality factor $Q$ associated to that resonance 


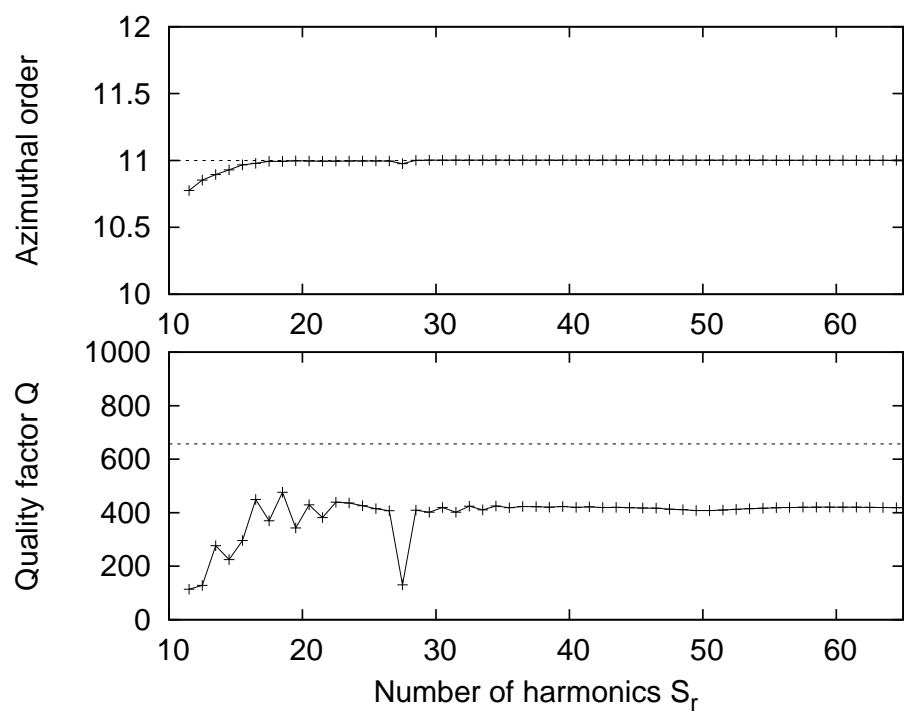

Figure 4. The convergence of the calculation of the quasi-TM resonance with order $m=11$. The dashed line in the $\mathrm{Q}$ graph is the reference quality factor calculated by 3D-FDTD for this resonance.

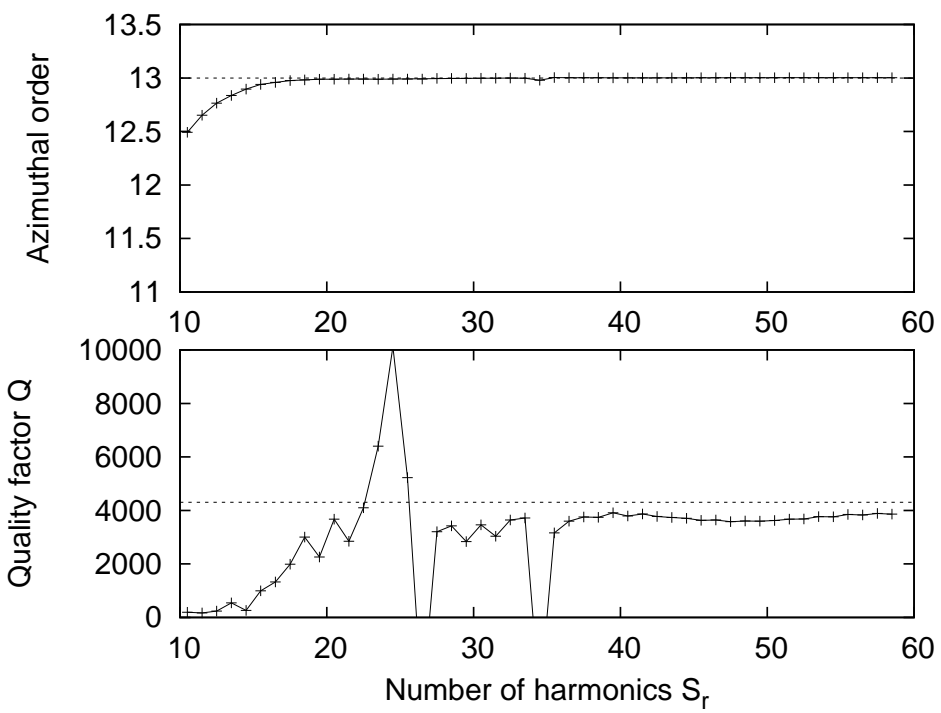

Figure 5. The convergence of the calculation of the quasi-TM resonance with order $m=13$. The dashed line in the $\mathrm{Q}$ graph is the reference quality factor calculated by 3D-FDTD for this resonance.

can be again determined from the complex effective index:

$$
Q=-\frac{\mathbb{R}\left\{n_{\mathrm{eff}}\right\}}{2 \mathbb{I}\left\{n_{\mathrm{eff}}\right\}}
$$

From a computational point of view, our implementation of the AFMM method in cylindrical coordinates has been written in $\mathrm{C}++$, interfacing with LAPACK and BLAS for matrix calculations. The main limitation of the 2D AFMM is due to the very large size of the matrices involved, which limits the number of harmonics which can be taken into account.

The first difficulty we have to face when treating the 2D case is to choose a correct size of the calculation window, by taking into account the shape of the waveguide core as well as its bending. This choice is a compromise between the necessity of representing a structure in sufficient detail while using a limited number of harmonics. 


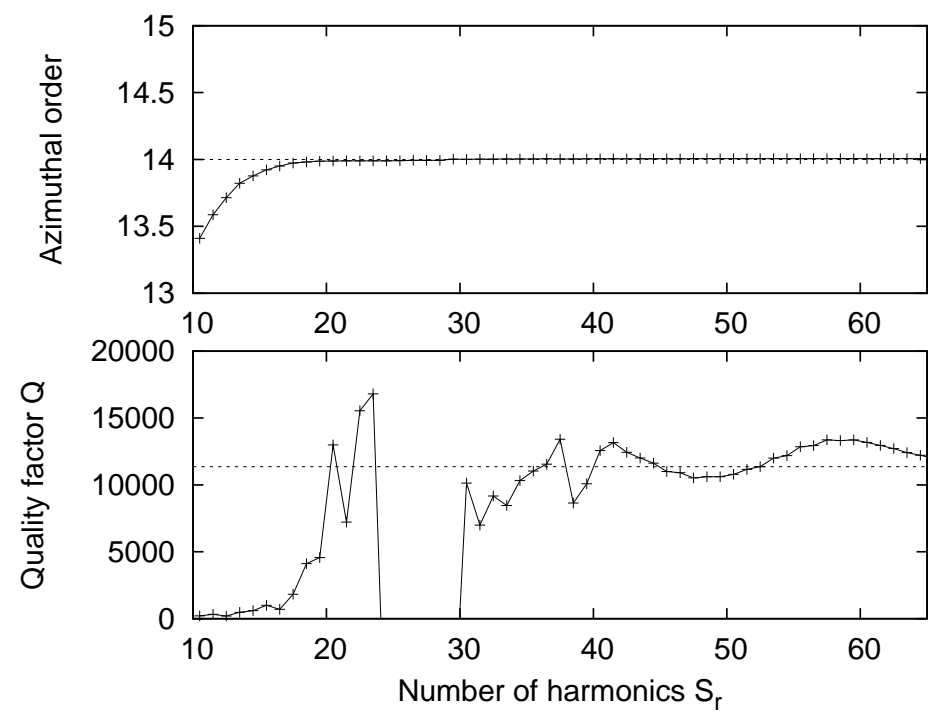

Figure 6. The convergence of the calculation of the quasi-TM resonance with order $m=14$. The dashed line in the $\mathrm{Q}$ graph is the reference quality factor calculated by 3D-FDTD for this resonance.

On the other hand, the calculation window must be wide enough to represent the evanescent fields out of the core of waveguides and leave enough place for PMLs. Since the waveguide will be lossy thanks to the bending, we need to carefully optimize the size of PML layers but, again, their size must be kept to a reasonable limit since they must be comprised inside the calculation window. The evaluation of high quality factors in resonators needs extremely efficient PMLs, since they must absorb the leaky field due to the bending. For our test case, we adopted a calculation window of $T_{r}=4 \mu \mathrm{m}$ and $T_{z}=2 \mu \mathrm{m}$. The wider range in the $r$ direction leaves more space to the wider PMLs, in order to absorb the field. For this reason, the total PML thickness was 1.5 $\mu \mathrm{m}$ in $r$ and $0.2 \mu \mathrm{m}$ in $z$ with a $\gamma$ coefficient of $0.5-\mathrm{j} 0.5$. A convergence study has been done in order to determine how many harmonics $S_{r}$ should be taken in the $r$ direction, while in the $z$ direction $S_{z}$ has been fixed to 15 .

Figure 4 describe the evolution of the calculated azimuthal order $m$ as well as the quality factor $Q$ for a quasi-TM resonance with $m=11$. It can be seen that a convergence is rapidly attained when the number of harmonics $S_{r}$ increases. The difference with the 3D-FDTD may be explained by taking into account the effect of the PMLs on the propagated mode, which is not very well confined. In figure 5 , is shown a result of the convergence study for the case of the quasi-TM resonance with azimuthal order $m=13$. When the number of harmonics taken in the $r$ direction is too small (less than 40), the behavior of the quality factor $Q$ is very unstable, but attains the convergence when the number of harmonics is sufficient. It can also be seen that the calculation of the azimuthal order attains a convergence much faster than the quality factor. In other words, from equations (22) and (23), we see that it is easier to calculate the real part than the imaginary part of the propagation constant. When the quality coefficient is higher (which is the case for quasi-TM resonance with $m=14$ ), it is more difficult to obtain a convergence, like it can be seen in figure 6 . This gives the practical limit of this method for determining very high quality factors.

Like what was done by Armaroli et al. ${ }^{16}$ we compared the resonance frequencies for quasi-TE and quasi-TM modes for several azimuthal orders. Table 3 shows a comparison between the quality factors of a quasi-TE mode with the values obtained by FDTD. Table 4 shows the same analysis performed for the quasi-TM case. From tables 3 and 4, it is evident that for a same azimuthal order an higher quality factor can be obtained from the quasi-TM mode, thanks to the size of the micro-disk which allows a better confinement for quasi-TM modes.

Figure 7 shows the calculated electric fields amplitudes for the fundamental quasi-TE mode in the micro disk at a frequency of $\lambda=1.1782 \mu \mathrm{m}$. The effect of the bending is visible by the fact that there is an evident shift of the field from the center of the waveguide core. The radiated field is also visible. The plotted amplitude of the field is arbitrary, but is coherent between the two field components. The discontinuities of the field on the $r$ axis are clearly evident for the preponderant $E_{r}$ field component. The fact that negative radii appear in the 


\begin{tabular}{clllll}
\hline order $m$ & 11 & 12 & 13 & 14 & 15 \\
\hline$\lambda$-FDTD & $1.2277 \mu \mathrm{m}$ & $1.1767 \mu \mathrm{m}$ & $1.1312 \mu \mathrm{m}$ & $1.0900 \mu \mathrm{m}$ & $1.0537 \mu \mathrm{m}$ \\
Q-FDTD $^{16}$ & 142 & 371 & 1081 & 2224 & 6224 \\
\hline$\lambda$-AFMM & $1.2310 \mu \mathrm{m}$ & $1.1782 \mu \mathrm{m}$ & $1.1319 \mu \mathrm{m}$ & $1.0910 \mu \mathrm{m}$ & $1.0532 \mu \mathrm{m}$ \\
$Q$-AFMM & 74 & 209 & 652 & 2047 & 6126 \\
\hline
\end{tabular}

Table 3. Comparison between the quality factors obtained by 3D-FDTD and by the AFMM method for quasi-TE resonances.

\begin{tabular}{|c|c|c|c|c|}
\hline order $m$ & 11 & 12 & 13 & 14 \\
\hline$\lambda$-FDTD & $1.3344 \mu \mathrm{m}$ & $1.2769 \mu \mathrm{m}$ & $1.2248 \mu \mathrm{m}$ & $1.1770 \mu \mathrm{m}$ \\
\hline$Q-\mathrm{FDTD}^{16}$ & 657 & 1916 & 4303 & 11369 \\
\hline$\lambda$-AFMM & $1.3402 \mu \mathrm{m}$ & $1.2816 \mu \mathrm{m}$ & $1.2285 \mu \mathrm{m}$ & $1.17929 \mu \mathrm{m}$ \\
\hline$Q$-AFMM & 419 & 1238 & 3848 & 12856 \\
\hline
\end{tabular}

Table 4. Comparison between the quality factors obtained by 3D-FDTD and by the cylindrical AFMM method for quasiTM resonances, with $S_{r}=55$.

calculation window does not have an influence on the calculations since they are inside the PML and there is almost no field in those regions.

The comparison between the resonating wavelength obtained by our version of AFMM and the published results for 3D-FDTD shows that our method can be effectively used to evaluate the resonance behavior of high confinement, low bending radius ring resonator. Our implementation provides an estimation of the resonance wavelengths which are rather accurate in comparison with the 3D-FDTD results, albeit tending to slightly under-estimate the quality factor. This is probably due to the effect of PMLs which are the sole source of field absorption in our calculation.

\section{CONCLUSION}

In this work, the Aperiodic Fourier Modal Method has been developed in cylindrical coordinates in order to study bent waveguides. We discussed the implementation of a full vectorial mode solver able to determine the propagation modes of a structure having an arbitrary cross-section and a fixed curvature radius. The method has been first tested in $1 \mathrm{D}$ and then applied to the full vectorial $2 \mathrm{D}$ case. The first tests in $1 \mathrm{D}$ have shown a satisfactory agreement of our technique with a semi-analytical analysis as well as the conformal mapping technique applied to a few test cases. For the 2D case, we applied the cylindrical coordinates AFMM to the study of the resonance properties in the near infrared of a high confinement micro ring made by silicon immersed in silica. The results have shown a good agreement with 3D-FDTD studies reported in the literature.

As a perspective of this work, in order to increase the ability to absorb the field leaking from waveguides, the study of asymmetric PMLs can be fruitful. This would allow to reduce the total size of the calculation
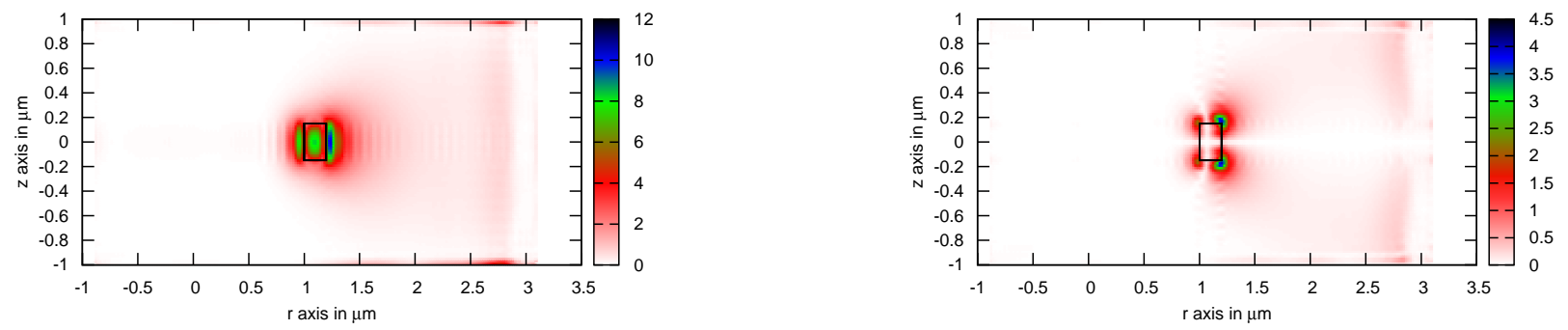

Figure 7. Module of the $r$ (on the left) and $z$ (on the right) components of the electric field for the quasi-TE fundamental mode at a wavelength of $\lambda=1.1782 \mu \mathrm{m}$, calculated by AFMM with $S_{r}=46$ and $S_{z}=15$. 
window, thus allowing a more precise representation of the structure in the Fourier space, with a fixed number of harmonics.

The main advantage of the AFMM over other methods is that it does not need a zero finding routine to determine the propagation constants of the structure. The eigenspace resolution leads directly to a set of guided modes, a discrete set of radiated modes and a discrete set of evanescent modes. Moreover, the cylindrical formulation is far much interesting than the conformal one. Indeed, considering a set of sections along the propagation ( $\theta$-axis here) as described by Lalanne and Silberstein, ${ }^{1,2}$ this method can be used to simulate the propagation through a structure designed by either straight or bent waveguides. The conformal method needs a resampling of the field from one section to the other whereas the cylindrical method does not need it. This would lead to a complete tool of guided propagation.

\section{ACKNOWLEDGMENTS}

The authors want to thank Pierre Benech for fruitful discussions.

\section{REFERENCES}

[1] Lalanne, P. and Silberstein, E., "Fourier-modal methods applied to waveguide computational problems," Optics Letters 25(15), 1092-1094 (2000).

[2] Silberstein, E., Lalanne, P., Hugonin, J., and Cao, Q., "Use of grating theories in integrated optics," Journal of the Optical Society of America A 18(11), 2865-2875 (2001).

[3] Li, L., "Recent advances and present limitations of the electromagnetic theory of diffraction gratings," Diffractive Optics and Micro-Optics (2000).

[4] Gray, R., [Toeplitz and circulant matrices: A review], Now Pub (2006).

[5] Lalanne, P. and Morris, G. M., "Highly improved convergence of the coupled-wave method for tm polarization," Journal of the Optical Society of America A 13(4), 779-784 (1996).

[6] Li, L., "Use of Fourier series in the analysis of discontinuous periodic structures," Journal of the Optical Society of America A 13, 1870-1876 (1996).

[7] Lalanne, P., "Improved formulation of the coupled-wave method for two-dimensional gratings," Journal of the Optical Society of America A 14(7), 1592-1598 (1997).

[8] Hewlett, S. and Ladouceur, F., "Fourier decomposition method applied to mapped infinite domains: scalar analysis of dielectric waveguides down to modal cutoff," Lightwave Technology, Journal of 13(3), 375-383 (1995).

[9] Hugonin, J. and Lalanne, P., "Perfectly matched layers as nonlinear coordinate transforms: a generalized formalization," J. Opt. Soc. Am. A 22 (september 2005).

[10] Hugonin, J. P. e. a., "Fourier modal methods for modelling optical dielectric waveguides," Optical and quantum electronics 37(1) (2005).

[11] Hiremath, K., Hammer, M., Stoffer, R., Prkna, L., and Čtyrokỳ, J., "Analytic approach to dielectric optical bent slab waveguides," Optical and Quantum Electronics 37(1), 37-61 (2005).

[12] Heiblum, M. and Harris, J., "Analysis of curved optical waveguides by conformal transformation," IEEE Journal of Quantum Electronics 11(2), 75-83 (1975).

[13] Martin, B., Morand, A., Benech, P., Leblond, G., Blaize, S., Lerondel, G., Royer, P., Kern, P., and Le Coarer, E., "Design of a compact static Fourier transform spectrometer in integrated optics based on a leaky loop structure," Optics Letters 34(2), 184-186 (2009).

[14] Kuwata-Gonokami, M., Jordan, R., Dodabalapur, A., Katz, H., Schilling, M., Slusher, R., and Ozawa, S., "Polymer microdisk and microring lasers," Optics Letters 20(20), 2093-2095 (1995).

[15] Chu, S., Little, B., Pan, W., Kaneko, T., Sato, S., and Kokubun, Y., "An eight-channel add-drop filter using vertically coupled microring resonators over a cross grid," IEEE Photonics Technology Letters 11(6), 691-693 (1999).

[16] Armaroli, A., Morand, A., Benech, P., Bellanca, G., and Trillo, S., "Three-dimensional analysis of cylindrical microresonators based on the aperiodic Fourier modal method," Journal of the Optical Society of America A 25(3), 667-675 (2008). 